\title{
Testing personalized medicine: patient and physician expectations of next-generation genomic sequencing in late-stage cancer care
}

\author{
Fiona A Miller ${ }^{\star}, 1,2$, Robin Z Hayeems ${ }^{1}$, Jessica P Bytautas ${ }^{1}$, Philippe L Bedard ${ }^{3}$, Scott Ernst ${ }^{4}$, Hal Hirte ${ }^{5}$, \\ Sebastien Hotte ${ }^{5}$, Amit $\mathrm{Oza}^{3}$, Albiruni Razak ${ }^{3}$, Stephen Welch ${ }^{4}$, Eric Winquist ${ }^{4}$, Janet Dancey ${ }^{6}$ and \\ Lillian L Siu ${ }^{3}$
}

Developments in genomics, including next-generation sequencing technologies, are expected to enable a more personalized approach to clinical care, with improved risk stratification and treatment selection. In oncology, personalized medicine is particularly advanced and increasingly used to identify oncogenic variants in tumor tissue that predict responsiveness to specific drugs. Yet, the translational research needed to validate these technologies will be conducted in patients with late-stage cancer and is expected to produce results of variable clinical significance and incidentally identify genetic risks. To explore the experiential context in which much of personalized cancer care will be developed and evaluated, we conducted a qualitative interview study alongside a pilot feasibility study of targeted DNA sequencing of metastatic tumor biopsies in adult patients with advanced solid malignancies. We recruited 29/73 patients and 14/17 physicians; transcripts from semi-structured interviews were analyzed for thematic patterns using an interpretive descriptive approach. Patient hopes of benefit from research participation were enhanced by the promise of novel and targeted treatment but challenged by non-findings or by limited access to relevant trials. Family obligations informed a willingness to receive genetic information, which was perceived as burdensome given disease stage or as inconsequential given faced challenges. Physicians were optimistic about long-term potential but conservative about immediate benefits and mindful of elevated patient expectations; consent and counseling processes were expected to mitigate challenges from incidental findings. These findings suggest the need for information and decision tools to support physicians in communicating realistic prospects of benefit, and for cautious approaches to the generation of incidental genetic information.

European Journal of Human Genetics (2014) 22, 391-395; doi:10.1038/ejhg.2013.158; published online 17 July 2013

Keywords: personalized medicine; cancer care; qualitative research; clinical trials; genetic testing; clinical sequencing

\section{INTRODUCTION}

Developments in genomics, including low-cost next-generation sequencing technologies, are expected to enable a more personalized approach to clinical care, with improved risk stratification and treatment selection. ${ }^{1,2}$ In oncology, personalized medicine is particularly advanced, with increased use of genomic testing technologies to identify those oncogenic variants in tumor tissue that predict responsiveness to specific drugs. ${ }^{3-5}$ Indeed, clinical leaders predict an 'evolution from non-specific cytotoxic drugs that damage both tumor and normal cells to more specific agents and immunotherapy approaches to produce greater effectiveness with less toxicity. ${ }^{4}$

Yet, extensive translational research is needed to validate promising biomarkers and demonstrate clinical and comparative effectiveness. ${ }^{6-8}$ Such research will identify results of variable clinical significance and may also be used to generate information about inherited health risks. ${ }^{9-12}$ Further, in the research context and in the early stages of translation to clinical care, these results will be generated in patients with late-stage disease, where the value of hope is enhanced and patients face complex decisions about the pursuit of small but risky benefits. ${ }^{13-16}$ Thus, delivering on the promise of personalized medicine raises questions about how best to address the needs of patients and their providers in the design and delivery of genomebased diagnostics. To date, however, social science research exploring the experiences of patients and physicians provides only partial insight.

Given the interest in and enthusiasm about personalized medicine, there is comparatively little empirical research reporting on the expectations and experiences of patients and providers who participate in such initiatives. In the context of personalized cancer care, much of the extant literature explores gene-expression profiling in the adjuvant and neo-adjuvant breast cancer setting (eg, Oncotype DX, Mammaprint). ${ }^{17-20}$ Findings from this literature point to broad enthusiasm about the potential to 'personalize' care 17,21,22 $^{\text {and }}$ reduce uncertainty. ${ }^{20,23}$ There is also, however, some evidence of patient confusion regarding the meaning of test results (eg, whether germline or somatic variants are identified). ${ }^{17}$ In addition, issues of

${ }^{1}$ Institute of Health Policy, Management and Evaluation, University of Toronto, Toronto, ON, Canada; ${ }^{2}$ Joint Centre for Bioethics, University of Toronto, Toronto, ON, Canada; ${ }^{3}$ Princess Margaret Cancer Centre-University Health Network, Toronto, ON, Canada; ${ }^{4}$ London Health Sciences Centre, London, ON, Canada; ${ }^{5}$ Juravinski Cancer Centre, Hamilton, ON, Canada; ${ }^{6}$ Ontario Institute for Cancer Research, Toronto, ON, Canada

*Correspondence: Dr FA Miller, Institute of Health Policy, Management and Evaluation, University of Toronto, 155 College St, 4th Floor, Toronto, ON M5T 3M6, Canada. Tel: + 416978 3703; Fax: + 416978 7350; E-mail: fiona.miller@utoronto.ca

Received 28 March 2013; revised 7 June 2013; accepted 21 June 2013; published online 17 July 2013 
access and equity have started to be raised in the conceptual literature, ${ }^{24}$ and there is some empirical evidence of consumer concern about access to relevant tests. ${ }^{17,21}$

In addition, emerging literature attends to the identification of genetic risk information using next-generation sequencing technologies and highlights expectations related to research participation and preferences for results, ${ }^{25}$ including incidental results. Yet, although research exploring patient experiences with testing for inherited health risks is extensive, ${ }^{26,27}$ research on the effects of receiving unexpected or uncertain genetic information is less developed. Available evidence indicates that genetic test results can be conveyed with limited psychological harm to individuals known to be at high risk, ${ }^{28-31}$ and highlights the importance of familial obligations in individuals' decisions to pursue such testing. ${ }^{32-35}$ However, psychosocial research also identifies the potential for a negative impact, where individuals undergo cancer genetic testing in the absence of a suggestive family or personal history. ${ }^{36}$

\section{MATERIALS AND METHODS}

We conducted a qualitative substudy embedded within a multicenter clinical feasibility study of genomic sequencing of tumor biopsies. The tumor biopsy study evaluated whether high-throughput sequencing, accompanied by verification of results in a clinical laboratory, was feasible, could be used as a clinical tool to guide cancer therapy and would add value to the information generated by traditional genotyping methods. ${ }^{37}$

With ethics approval from the Ontario Cancer Research Ethics Board, University of Toronto, and participating hospitals, as well as informed consent from participants, we conducted open-ended, semi-structured interviews from May 2011 to June 2012 with (i) adult patients with advanced solid malignancies enrolled in the tumor biopsy study; to enable face-to-face interviews, only patients from the three southern Ontario sites were eligible; and (ii) medical oncologist investigators involved in recruitment for the study at the three southern Ontario and two northern Ontario sites.

Patients with poor performance status or psychosocial distress, those residing distant from the Greater Toronto Area or who were non-conversant in English were excluded. Physicians approached patients about participating in the interview study at the meeting at which they reviewed the results of genome sequencing. JPB or FAM contacted willing participants within 2-4 weeks to arrange an interview in the patient's home or community, although some interviews were conducted during a clinic visit. Physicians were invited by JPB or FAM to participate in a semi-structured interview regardless of whether their patients participated in the interview study; interviews were conducted using phone or in person during regular office hours.

With the use of a semi-structured interview guide, we explored patients' and physicians' experiences with study enrollment, the tumor testing process, result interpretation (whether positive, negative or none) and expectations on how these results might inform care. The first phase of the tumor biopsy study focused on a limited panel of oncogenes; hence no clinically relevant inherited risk information was generated or disclosed. The next phase of the study used an expanded platform in which incidental inherited risk information is generated and could be confirmed and reported. Thus, to inform future practice, we probed attitudes toward the possibility of receiving inherited risk information as an incidental outcome of genomic analysis.

To explore commonality or divergence in the expectations of patients and physicians, we searched for and compared shared themes across the two groups (see Table of Supplementary quotes) and adopted a qualitative interpretive descriptive' approach to support rich description and low-inference interpretations. ${ }^{38,39}$ Specifically, taped interview data were transcribed verbatim and stored and managed electronically. Data were analyzed using the techniques of constant comparison adapted from grounded theory to search for thematic patterns and relations..$^{40,41}$ We used a mixed strategy for coding, using predetermined codes from our interview guide and allowing codes to emerge empirically from the data.

\section{Descriptive results}

Twenty-nine of the seventy-three patients enrolled in the tumor biopsy study at three sites participated in the interview study. Some patients were excluded by their physician because of poor performance status $(n=21)$ or psychosocial distress $(n=1)$, limited English language $(n=5)$ or distant residence $(n=2)$; four patients declined to participate, eight were lost to follow-up after agreeing to participate (two of whom withdrew because of impending death), one patient died before their physician could approach them about the substudy and two were not approached after their tumor analysis failed because of insufficient sample.

The majority of patients who participated in the interview were female, with both common (breast, ovarian, colorectal and lung) and more rare cancers. Eleven of twenty-nine patients harbored a mutation that was potentially relevant to therapeutic decisions (Table 1) and many had subsequently been referred to a relevant clinical trial. Patients who completed the qualitative interview did not differ markedly from the full population of potentially eligible patients; we cite patient respondents by ID number, using the prefix PT, and indicate whether or not the individual was identified as harboring a somatic mutation potentially relevant to therapeutic decisions (ie, reported mutation and no mutation reported). Fourteen of seventeen eligible physicians ( $100 \%$ from four of the five sites) participated in qualitative interviews (cited by ID number, with the prefix DR) (Table 1).

\section{Qualitative results}

We review patient and physician expectations across three themes: (i) motivations for study participation; (ii) interpretations of test results; and (iii) anticipations for inherited risk information.

\section{Motivations for study participation}

Patients. Although many patients hoped that their participation in research might advance science, the potential for personal benefit drove participation: ...that's why I initially got into that one study. I was feeling really down. They offered some type of hope, you know? And so, that's why you sign up. Maybe there is a fit. Who knows? And you grasp at anything, I think. [PT-20-reported mutation]

The potential of genome sequencing was perceived as an added source of hope. These technologies were attractive as novel or 'cutting edge,' or because they seemed to offer targeted and more effective therapy:

When the biopsy study came up what I really was attracted to about it was that for the type of tumour I have there's really nothing proven; anything we would do is just a stab in the dark. It was a genetic study that might show, you know, a treatment option that would be successful for me. [PT-37-reported mutation]

\section{Table 1 Eligible patients}

\begin{tabular}{lcc} 
& $\begin{array}{c}\text { Participated in interview } \\
(\mathrm{n}=29)\end{array}$ & $\begin{array}{c}\text { Did not participate } \\
(\mathrm{n}=44)\end{array}$ \\
\hline Age (average) & 57 & 58 \\
Age (range) & $41-80$ & $31-80$ \\
Gender (proportion female) & $17(59 \%)$ & $25(57 \%)$ \\
& & \\
Cancer type & & $4(9 \%)$ \\
Breast & $7(24 \%)$ & $8(18 \%)$ \\
Colon/colorectal & $4(14 \%)$ & $2(5 \%)$ \\
Lung & $2(7 \%)$ & $8(18 \%)$ \\
Ovarian & $4(14 \%)$ & $3(7 \%)$ \\
Prostate & $2(7 \%)$ & $19(43 \%)$ \\
Other & $10(34 \%)$ & $14(32 \%)^{a}$ \\
Observed mutations (proportion with) & $11(38 \%)$ &
\end{tabular}

aTwo samples were insufficient for mutation analysis. 
Physicians. Providers expressed awareness of the hope driving their patients' willingness to participate in research and the need to not oversell the study's potential.

... in oncology...patients accept single digit percentages of benefit...I think it's a human behavioural thing about hope and that we take risks when we're faced with something that's life threatening... [DR-85]

Physicians perceived the study as important, to remain 'cutting edge' and explore the potential of a paradigm shift in cancer care; they also hoped it might provide some patients with treatment options.

It's definitely a new frontier because we're looking at gaining some molecular information about the patient's disease at the time that the therapeutic decision is needed. [...] I think this is in some ways a paradigm shift in our thinkinginstead of approaching it as breast cancer or lung cancer, you know, we're really trying to understand... what the molecular mechanisms are that may be driving the cancer and to match the therapy to it. [DR-30]

\section{Interpreting the results of genome sequencing}

Patients. Patients were hopeful about any genome sequencing results that were identified and perceived special promise in trials that were recommended as a result:

They found some mutations, which is untypical for my kind of cancer, but it is very well studied for other cancers. And therefore there are actually three options. Currently one trial is open right now and it looks the most promising. [...] As far as I understand it, it is my best hope. [PT-11-reported mutation] Patients expressed some willingness to expend more energy to pursue trials, 'when we've got this specific information.' [PT-25-reported mutation] Correspondingly, they perceived challenges when such trials were not-or not readily-available. Offered the option of traveling across the province for a relevant clinical trial, this patient noted:

That's not an option. I'm not going to travel to the [hospital]. Just it's already too tiring...my body has suffered a lot in those last 6 years. And there comes a point in time that I have to say, 'Enough is enough.' [PT-23-reported mutation]

Some patients in whom no mutation was identified expressed clear disappointment, whereas others were philosophical in facing perceived setbacks.

Look, it showed me that one possible option for treatment isn't available. And, so, with that information I now know, ok, that's not an option. I've ticked it off my list. So now I need to search elsewhere. And I think that's an important piece of information. Whether it was positive or negative. [PT-19-no mutation reported]

Physicians. Physicians acknowledged some personal gratification when they were able to tell the patient 'well, this is what was found and this is what we're going to do' [DR-59] and some patient disappointment when no mutations were identified:

So, in many cases, you explain to them, 'Unfortunately, we haven't found anything.' And, I think there's a certain element of disappointment for them... not so much that they've gone through it and it's been a waste of time, but there's really a hope that we'll find something that will potentially open up more options for them that may improve their outcome. [DR-30]

In addition, physicians acknowledged several limitations with the results of testing, as a limited number of genes were analyzed and clinical utility was not established. Further, many expressed concern at the actual or potential lack of accessible clinical trials for patients in whom a mutation was identified, as

'there are very real limits and a shortage of targeted agents that we might be able to utilize.' [DR-65]

Discussing one difficult case, this physician reflected on the challenges patients faced in deciding what burdens to endure in pursuing the small chance of benefit:

There was some indication that in [city] they had a particular drug that may work, but for her the biggest thing is that she has three young kids... for her to now have to try to decide to do treatment in some place other than [city] because potentially there's a drug out there with some preclinical information that may work for her is a tough decision. [DR-42]

\section{Anticipating inherited risk information}

Patients. All patients except two expressed a willingness to receive incidental genetic information about inherited health risks if it were generated through genome sequencing for cancer treatment. Patients pondered the value of this information for its relevance within families and as cancer patients struggling with a life-threatening disease.

Considering this as genetic information, most patients anticipated that it could provide important insight and that they should pursue it for its value to family members.

I would like that... to know that there is something else in my family, anything else. I have two children, so I would like to know if there is something. [PT-13-no mutations reported]

Considering this information as pertaining to persons with terminal cancer, even those patients willing to receive such information reflected on it as an additional burden. For many, such a burden was a great deal to bear at a difficult time in life:

One thing at a time. Just fighting cancer is hard enough never mind being told now you're going to be fighting this or fighting that. And after doing this for 4 years, I mean, just let's deal with this... [PT-18-reported mutation]

For others, however, the challenge posed by cancer seemed so immense that additional bad news seemed insignificant by comparison:

At this point, like, so what? That would be the least of my issues. If you can deal with [cancer], you can deal with anything. [PT-38-reported mutation]

Physicians. Physicians anticipated that their patients would value inherited risk information because of its relevance for family members:

I think most patients want to know about that kind of information-you know, if they're carrying a germline mutation... [DR-85]

Some also pondered the significance of generating such information in patients with advanced cancer-seeing this as secondary in importance for patients with so much on their plate:

For the most part that's not a big part of what they're asking. They're really asking, 'how is this going to influence my care right now?' I think [for] most patients it's enough already to deal with the fact that they have an incurable disease with limited options in terms of therapy. [DR-30]

Physicians were quick to identify the need for informed consent and genetic counseling to support patients in considering and managing the receipt of such information.

You know if people are informed about [potential germline findings], then that's kind of their decision to pursue that. People are not mandated to confirm that something is in the germline... So, I think it needs to be explained upfront in the consent... [DR-42]

\section{DISCUSSION}

This qualitative study explored patient and physician expectations of genome sequencing alongside a clinical study that assessed the feasibility of using this technology. We interviewed patients with advanced solid malignancies enrolled in the clinical study, some of whom had received information about an oncogenic mutation that might be clinically actionable. We also interviewed the physicians who recruited patients into the clinical study, reported results and sought to find treatment options. The gravity of the situation facing our participants was apparent in our recruitment rates. Of the 73 potentially eligible patients, 21 were excluded because of poor performance status and at least two others died before they could be recruited or interviewed.

The context of life-threatening disease informed all that we learned from participants. As is well-known from literature on clinical trials, ${ }^{13-16}$ patients enrolled themselves in research because they were out of options and valued the hope that experimental approaches might offer. Altruistic motivations were also present, ${ }^{8}$ but this was in addition to and not instead of the hope for personal gain. ${ }^{42}$ Yet, although patients were like other hopeful participants in 
clinical trials, the promise of genomics enhanced their expectations of benefit. ${ }^{17,26,27}$ Patients believed that genomic information about their cancer held particular promise because it was novel and offered the potential of targeted therapeutics. Patients who received information about tumor mutations were especially hopeful of the experimental therapeutics these test results made relevant, and disappointed when suitable clinical trials were not available. Patients who did not receive such results were also disappointed; yet, consistent with the hope that had motivated their participation, patients were also pragmatic in continuing their quest for therapeutic options.

Providers were similarly motivated by the cutting-edge promise of genomic science, but their hopes were less acute and their concerns were more practical than those expressed by patients. Physicians believed that developments in genomics might yield benefits for patients, but they expected most of these benefits to be realized in the future. They valued the hope that the study might offer some patients, and also acknowledged the delicate balance required when navigating patient hopes and vulnerabilities in this context. In addition, and unlike patients, physician respondents acknowledged the limitations of testing for clinical decision making and highlighted the very real challenges of access to the experimental therapeutics suggested by genomic markers, as the personalized approach to cancer care is developed and tested. ${ }^{24}$

The context of late-stage cancer care also conditioned the prospect of receiving and reporting incidental inherited risk information. Patient participants experienced this prospect as cancer patients for whom this was either 'more bad news' or a seemingly insignificant addition to an already overflowing plate. At the same time, patients experienced the prospect as specifically family information and did so in ways that align with findings from an expansive body of research on genetic testing. ${ }^{28-31,36}$ With few exceptions, patients expected genetic knowledge to be useful, permitting action to avert harm. Further, these potential benefits were contemplated by patients as members of family networks, for which they bore personal responsibilities to gain and convey relevant risk information. ${ }^{43,44}$ Provider participants endorsed the receipt of incidental risk information as important but secondary to the issues at hand. They acknowledged the psychosocial-, family- and privacy-related complexities brought to bear by generating this information but anticipated that genetic counseling infrastructure could be harnessed to mitigate potential challenges.

\section{LIMITATIONS}

Several limitations to this study must be acknowledged. First, our patient sample comprises a subset of patients enrolled in the tumor biopsy study; however, few patients refused an interview, and participants and non-participants appear broadly similar. A second limitation arises from the retrospective nature of patient accounts regarding their initial expectations from the study; however, the fact that physicians' reflections about patient recruitment were consistent with patient reflections on their participation provides additional support for our interpretations. Finally, stated preferences regarding the hypothetical receipt of inherited risk information are unreliable; however, our analysis of participants' interpretive framework for considering this prospect is rich and insightful.

\section{CONCLUSION}

This study provides a rich interpretation of the experiential context in which much of personalized cancer care will be developed and evaluated. This is the context of hope for benefit, where chances of benefit are small. Such hope is not necessarily inappropriate or without intrinsic value, although physicians are mindful of the need to balance hope with realistic assessments of potential benefit and concerned at the challenge of access to matched experimental targeted therapies. This context also conditions expectations for the incidental identification of inherited health risks. Although patients anticipate its value, the context of late-stage cancer care can be a very hard place to get more 'bad news', and perceived family obligations might make its receipt very hard to refuse. These findings suggest the need for information and decision tools to support physicians in communicating realistic prospects of benefit, and for cautious approaches for the generation of incidental genetic information. ${ }^{12}$

\section{CONFLICT OF INTEREST}

The authors declare no conflict of interest.

\section{ACKNOWLEDGEMENTS}

We thank the Ontario Institute for Cancer Research for funding and our physician participants for sharing time and expertise. We also thank the patients who, despite being gravely ill, gave generously of their time to share their experiences.

1 Hamburg M, Collins F: The path to personalized medicine. N Engl J Med 2010; 363: 301-304.

2 Chan I, Ginsburg G: Personalized medicine: progress and promise. Annu Rev Genomics Hum Genet 2011; 12: 217-244.

3 Borden E, Raghavan D: Personalizing medicine for cancer: the next decade. Nat Rev Drug Discov 2010; 9: 343-344.

4 Schlisky R: Personalized medicine in oncology: the future is now. Nat Rev Drug Discov 2010; 9: 363-366.

5 Roychowdhury S, lyer M, Robinson D et al: Personalized oncology through integrative high-throughput sequencing: a pilot study. Sci Trans/ Med 2011; 3: 111ra21.

6 Wideroff L, Phillips K, Randahawa G et al: A health services research agenda for cellular, molecular and genomic technologies in cancer care. Public Health Genomics 2009; 12: 233-244.

7 Kwon S, Cho H, Choi J, Jee B, Jo Y, Woo H: Perspectives of integrative cance genomics in next generation sequencing era. Genomics Inform 2012; 10: 69-73.

8 Facio F, Brooks S, Loewenstein J, Green S, Biesecker L, Biesecker B: Motivators for participation in a whole-genome sequencing study: implications for translationa genomics research. Eur J Hum Genet 2011; 19: 1213-1217.

9 Kohane E, Hsing M, Kong S: Taxonomizing, sizing and overcoming the incidentalome. Genet Med 2012; 14: 399-404.

10 Ku C-S, Wu M, Cooper D et al: Technological advances in DNA sequence enrichment and sequencing for germline genetic diagnosis. Expert Rev Mol Diagn 2012; 12: 159-173.

11 Green RC, Berg JS, Grody WW et al: ACMG recommendations for reporting of incidental findings in clinical exome and genome sequencing. Genet Med 2013, 15: 565-574.

12 van El C, Cornel M, Borry P et al: Whole-genome sequencing in health care: recommendations of the European Society of Human Genetics. Eur J Hum Genet 2013; 21: 580-584.

13 Matsuyama R, Reddy S, Smith T: Why do patients choose chemotherapy near the end of life? A review of the perspective of those facing death from cancer. $J$ Clin Oncol 2006; 24: 3490-3496.

14 McClement S, Chochinov H: Hope in advanced cancer patients. Eur J Cancer 2008, 44: 1169-1174.

15 Sulmasy D, Astrow A, He M et al: The culture of faith and hope: patients' justifications for their high estimations of expected therapeutic benefit when enrolling in early phase oncology trials. Cancer 2010; 116: 3702-3711.

16 Wootten A, Abbott J, Siddons H, Rosenthal M, Costello A: A qualitative assessment of the experience of participating in a cancer-related clinical trial. Support Care Cancer 2011; 19: 49-55.

17 Pelligrini I, Rapti M, Extra J-M et al: Tailored chemotherapy based on tumour gene expression analysis: breast cancer patients' misinterpretaitons and positive attitudes. Eur J Cancer Care 2012; 21: 242-250.

18 Bueno-de-Mesquita J, van Harten W, Retel V et al: Use of 70-gene signature to predict prognosis of patients with node-negative breast cancer: a prospective community based feasibility study (RASTER). Lancet Oncol 2007; 8: 1079-1087.

19 Oratz R, Paul D, Cohn A, Sedlacek S: Impact of a commercial reference laboratory test recurrence score on decision making in early-stage breast cancer. J Oncol Pract 2007; 3: 182-287.

20 Lo S, Mumby P, Norton J et al: Prospective multicenter study of the impact of the 21-Gene Recurrence Score Assay on medical oncologist and patient adjuvant breast cancer treatment selection. J Clin Oncol 2010; 28: 1671-1676. 
21 Bonter K, Desjardins C, Currier N, Pun J, Ashbury F: Personalised medicine in Canada: a survey of adoption and practice in oncology, cardiology and family medicine. BMJ Open 2011; 1: e000110.

22 O'Neill S, Brewer N, Lillie S et al: Women's interest in gene expression analysis for breast cancer recurrence risk. J Clin Oncol 2007; 25: 4628-4634.

23 Andrykowski M, Burris J, Walsh E, Small B, Jacobsen P: Attitudes toward information about genetic risk for cognitive impairment after cancer chemotherapy: Breast cancer survivors compared with healthy controls. J Clin Oncol 2010; 28: 3442-3447.

24 McClellan K, Avard D, Simard J, Knoppers B: Personalized medicine and access to health care: potential for inequitable access? Eur J Hum Genet 2013; 21: 143-147.

25 Facio $\mathrm{F}$, Eidem $\mathrm{H}$, Fisher $\mathrm{T}$ et al: Intentions to receive individual results from whole-genome sequencing among participants in the ClinSeq study. Eur J Hum Genet 2013; 21: 261-265.

26 Issa A, Tufail W, Hutchinson J, Tenorio J, Poonam Baliga M: Assessing patient readiness for the clinical adoption of personalized medicine. Public Health Genomics 2009; 12: 163-169.

27 Gordon E, Griffin G, Wawak L, Pang H, Gollust S, Bernhardt B: "It's not like judgment day": public understanding of and reactions to personalized genomic risk information. $J$ Genet Couns 2012; 21: 432-432.

28 Schwartz M, Peshkin B, Hughes C, Main D, Isaacs C, Lerman C: Impact of BRCA1/BRCA2 mutation testing on psychologic distress in a clinic-based sample. J Clin Oncol 2002; 20: 514-520.

29 Lerman C, Narod S, Schulman $\mathrm{K}$ et al: BRCA1 testing in families with hereditary breast-ovarian cancer: a prospective study. JAMA 1996; 275: 1885-1892.

30 Croyle R, Smith K, Botkin J, Baty B, Nash J: Psychological responses to BRCA1 mutation testing: preliminary findings. Health Psychol 1997; 16: 63-72.

31 Broadstock M, Michie S, Marteau T: Psychological consequences of predictive genetic testing: a systematic review. Eur J Hum Genet 2000; 8: 731-738.

32 Hallowell N: Doing the right thing: genetic risk and responsibility. Sociol Health IIIn 1999; 21: 597-621.
33 Hallowell N, Foster C, Eeles R, Ardern-Jones A, Murday V, Watson M: Balancing autonomy and responsibility: the ethics of generating and disclosing genetic information. J Med Ethics 2003; 29: 74-83.

34 Hallowell N, Ardern-Jones A, Eeles R et al: Guilt, blame and responsibility: men's understanding of their role in the transmission of BRCA1/2 mutations within their family. Sociol Health IIIn 2006; 28: 969-988.

35 Etchegary H, Miller F, de Laat S, Wilson B, Carroll J, Cappelli M: Decision-making about inherited cancer risk: exploring dimensinos of genetic responsibility. J Genet Couns 2009; 18: 252-264.

36 Metcalfe K, Poll A, Royer R et al: Screening for founder mutations in BRCA1 and BRCA2 in unselected jewish women. J Clin Oncol 2010; 28: 387-391.

37 Tran B, Brown A, Bedard P et al: Feasibility of real time next generation sequencing of cancer genes linked to drug response: results from a clinical trial. Int J Cancer Supp 2013; 132: 1547-1555.

38 Sandelowski M: What's in a name? Qualitative description revisited. Res Nurs Health 2010; 33: 77-84.

39 Thorne S: Interpretive description. Walnut Creek, CA, USA: Left Coast Press, 2008.

40 Charmaz K: Constructivist and objectivist grounded theory; In: Denzin N, Lincoln Y editors. The SAGE handbook of qualitative research. Thousand Oaks, CA, USA: Sage, 2000.

41 Charmaz K: Constructing grounded theory: a practical guide through qualitative analysis. London, UK: Sage, 2006.

42 Hallowell N, Cooke S, Crawford G, Lucassen A, Parker M: Distinguishing research from clinical care in cancer genetics: theoretical justifications and practical strategies. Soc Sci Med 2009; 68: 2010-2017.

43 Forrest K, Simpson S, Wilson B et al: To tell or not to tell: Barriers and facilitators in family communication about genetic risk. Clin Genet 2003; 64: 317-326.

44 Wilson B, Etchegary H.: Family communication of genomic information; In: Teryack K editor. Handbook of genomics and the family: Psychosocial context for children and adolescents. New York, NY, USA: Springer, 2010.

Supplementary Information accompanies this paper on European Journal of Human Genetics website (http://www.nature.com/ejhg) 